\title{
Molecular outflows from G35.2-0.74N
}

\author{
J. R. Birks ${ }^{1}$, G. A. Fuller ${ }^{1}$, and A. G. Gibb ${ }^{2, \star}$ \\ 1 School of Physics \& Astronomy, University of Manchester, Sackville Street, PO Box 88, Manchester M60 1QD, UK \\ e-mail: joseph@josephbirks.com; G.Fuller@manchester.ac.uk \\ 2 Department of Astronomy, University of Maryland, College Park, MD 20742, USA
}

Received 8 April 2005 / Accepted 6 July 2006

\section{ABSTRACT}

\begin{abstract}
We present interferometric observations of the massive star-forming region associated with G35.2-0.74N using the Berkeley Illinois Maryland Association Array. With the aim of better understanding the outflow in this region we observed ${ }^{12} \mathrm{CO} J=1 \rightarrow 0, \mathrm{C}^{17} \mathrm{O} J=$ $1 \rightarrow 0$ and the $2.7 \mathrm{~mm}$ continuum. The $\mathrm{C}^{17} \mathrm{O}$ and continuum emission peak close to the sources G35.2-0.74N (G35.2N) and G35MM2 and indicate a mass of $\sim 40-140 M_{\odot}$ of circumstellar material associated with these sources. The ${ }^{12} \mathrm{CO}$ traces a weak filament of emission coincident with the radio and infrared jet from G35.2N but the emission is dominated by an extended outflow with a NE-SW axis which has a total mass of $\sim 13 M_{\odot}$. Each lobe of this extended outflow has a hollow shell structure and the location of these shells makes the source G35MM2 a more likely candidate for the source driving the outflow than G35.2N. The mass-velocity distribution is calculated for several parts of the outflow. Fitting these distributions with power laws some of the same break-points are seen as previously identified in the ${ }^{12} \mathrm{CO} J=3 \rightarrow 2$ emission from the outflow. We conclude this indicates the temperature dependence of emissivity is not responsible for all the break-points seen and molecular dissociation is a more plausible explanation for their origin. We model the molecular outflow using the ZEUS-2D hydrodynamic code which we have augmented so that it can also track the composition of the gas. We find that a general hydromagnetic wind, without an enchanced, on axis, jet-like component, can reproduce the shape of the observed outflow. Models looking at the time evolution of the stellar wind indicate that the structure of the outflow is dominated by the initial wind conditions, rather than its later evolution. The models also show that scaling the density of the wind profile effects the apparent collimation of the resulting outflow. This may help explain some of the apparent differences between outflows from high mass and low mass young stars.
\end{abstract}

Key words. ISM: jets and outflows - ISM: individual objects: G35.2-0.7N - radio lines: ISM

\section{Introduction}

Molecular outflows associated with low-mass stars are relatively well understood. It is generally thought that the interaction of the magnetic field with accreting material causes the ejection of high velocity, well collimated streams of matter (Königl \& Pudritz 2000). It is these jets, as they emerge in the polar direction, that form a bow-shock in the surrounding circumstellar medium and drive the formation of a dense expanding shell. This shocked shell of molecular gas is observable as the phenomenon known as a bipolar molecular outflow. In the case of high-mass star formation, however, this model runs into difficulty. Outflows are often associated with massive young stars but the associated jets are not: only a handful of jets are associated with $10^{4} L_{\odot}$ or more luminous sources (Garay \& Lizano 1999). The connection between jets and outflows in massive star formation is, therefore, less than evident.

The study of molecular outflows plays an important role in one of the ongoing debates within astrophysics, simply summarised as the question: do high-mass stars form in the same manner as low-mass stars? The fact that we see outflows in abundance across the mass spectrum does suggest a degree of similarity. However, massive outflows do bear some consistent differences to their low-mass counter-parts. Most obviously, they are more massive, often surprisingly so with some outflows made

* Present address: Department of Physics and Astronomy, University of British Columbia, 6224 Agricultural Rd, Vancouver, BC, V6T 1Z1, Canada; e-mail: agg@astro.ubc.ca up from hundreds of solar masses of material (see Churchwell 1997; and Beuther et al. 2002 for examples). Also, outflows from high mass sources appear to be less collimated (Richer et al. 2000), something which has been difficult to reproduce in models of jet-driven bow shocks. However Beuther et al. (2002) point out that the derivation of collimation values has a strong dependence on the angular resolution of the data. In their sample observed with $11^{\prime \prime}$ resolution, they find a mean collimation of 2.1 and predict this would be higher given data taken with higher resolution.

These differences between high and low-mass outflows (Richer et al. 2000) have lead to the examination of alternative models (e.g. Lee et al. 2001). In particular, models of a molecular shell driven by a wide-angle wind may show more promising topology for high mass outflows with the shell being less collimated than a jet-driven shell.

In order to fully understand massive outflows it is clear that high resolution data are necessary. In this paper we present interferometric maps of the massive star-forming region G35.2$0.74 \mathrm{~N}$ (hereafter G35.2N). This region is in the northern part of the cloud G35.2-0.74 (Little et al. 1983) at a distance of $2 \mathrm{kpc}$. The G35.2N region contains a mass of $\sim 500 M_{\odot}$ within about $0.3 \mathrm{pc}$ of the central IRAS source IRAS18556+0136 (Little et al. 1998). The region has a systemic velocity of $\sim 34 \mathrm{~km} \mathrm{~s}^{-1}$ and a linewidth of about $3 \mathrm{~km} \mathrm{~s}^{-1}$ in $\mathrm{C}^{18} \mathrm{O}$. The luminosity of the region and spectra of the infrared nebula in the region (Dent et al. 1985) imply the presence of an embedded B0.5 star. CO maps of this source by Dent et al. (1985), 
were interpreted as a single $\mathrm{CO}$ bipolar outflow lying almost in the plane of the sky (a low inclination angle), and at a position angle of $65^{\circ}(\mathrm{E}$ of $\mathrm{N})$. A more recent study of the ${ }^{12} \mathrm{CO} J=3 \rightarrow$ 2 emission by Gibb et al. (2003) has shown that the CO distribution is likely to be caused by multiple sources: potentially up to four separate outflows may make up the final picture. The presence of a N-S jet seen in the infra-red (Fuller et al. 2001) and the radio (Heaton \& Little 1988) appears to make this source a promising example of a coincidence between a massive outflow and a jet, even though a large precession angle would be involved. Gibb et al. (2003) have also detected a separate CO feature associated with the jet.

A large rotating interstellar disk-like structure lying almost perpendicular to the $\mathrm{CO}$ outflow was detected in ammonia emission by Dent et al. (1985). Gibb et al. (2003) (who also detected the disk in $\mathrm{H}^{13} \mathrm{CO}^{+}, \mathrm{H}^{13} \mathrm{CN}$ and the $3.5 \mathrm{~mm}$ continuum) reinterpreted it as a rotating, flattened envelope which is fragmented in nature and may contain a number of YSOs. A new deeply embedded millimetre source, named G35MM2, lies in this disk/envelope, but little is known about this new source (Gibb et al. 2003).

G35.2N is a well studied region, though there are still questions that have no satisfactory answer. The high resolution data presented in this paper help address some of these uncertainties and provide important insight into the workings of massive outflows and massive star formation in general.

\section{Observations}

The data presented here were obtained using the Berkeley Illinois Maryland Association (BIMA) Array in three different configurations: D (the shortest baselines), $\mathrm{C}$ and $\mathrm{B}$ (the longest baselines). The $\mathrm{D}$ and $\mathrm{C}$ array data consist of a 7 field mosaic in a hexagonal formation while the $\mathrm{B}$ array data are made up of only a single field as a trade-off between sensitivity (lower, due to the smaller synthesised beam) versus sky coverage and consistency with data from other array configurations. The phase calibrator 1751+096 (assuming a flux of 3.1 Jy) was observed periodically throughout each track. The correlator was set up to simultaneously observe ${ }^{12} \mathrm{CO} J=1 \rightarrow 0$ with a bandwidth and spectral resolution of $50 \mathrm{MHz}$ and $391 \mathrm{kHz}\left(1.03 \mathrm{~km} \mathrm{~s}^{-1}\right)$ and the $\mathrm{C}^{17} \mathrm{O} J=1 \rightarrow 0$ transition with a bandwidth and spectral resolution of $25 \mathrm{MHz}$ and $98 \mathrm{kHz}$. The remaining bandwidth was used to observe the $2.7 \mathrm{~mm}$ continuum. The data were calibrated and reduced using the normal procedures within MIRIAD. The ${ }^{12} \mathrm{CO}$ images shown here were produced weighting given by setting robust $=0.25$ and de-convolved from the dirty synthesised beam using the task MOSMEM (unless otherwise stated). The range of UV distances in the data means we are not sensitive to scales larger than about $1^{\prime}$. The $\mathrm{C}^{17} \mathrm{O}$ and continuum data were weighted so only the shortest baselines (equivalent to D array) contributed as the addition of the longer spacings concealed the detection with noise. The pointing centre of the centre field of the mosaic was RA $(\mathrm{J} 2000)=18^{\mathrm{h}} 58^{\mathrm{m}} 12.93^{\mathrm{s}}$, $\operatorname{Dec}(\mathrm{J} 2000)=+01^{\circ} 40^{\prime} 39.50^{\prime \prime}$.

\section{Results}

\subsection{CO emission}

Figure 1 shows the integrated CO emission while Fig. 2 shows the blue and red-shifted emission. These maps are dominated by a NE-SW oriented outflow, consistent with previous $\mathrm{CO}$ observations of the region. However it is clear that the higher

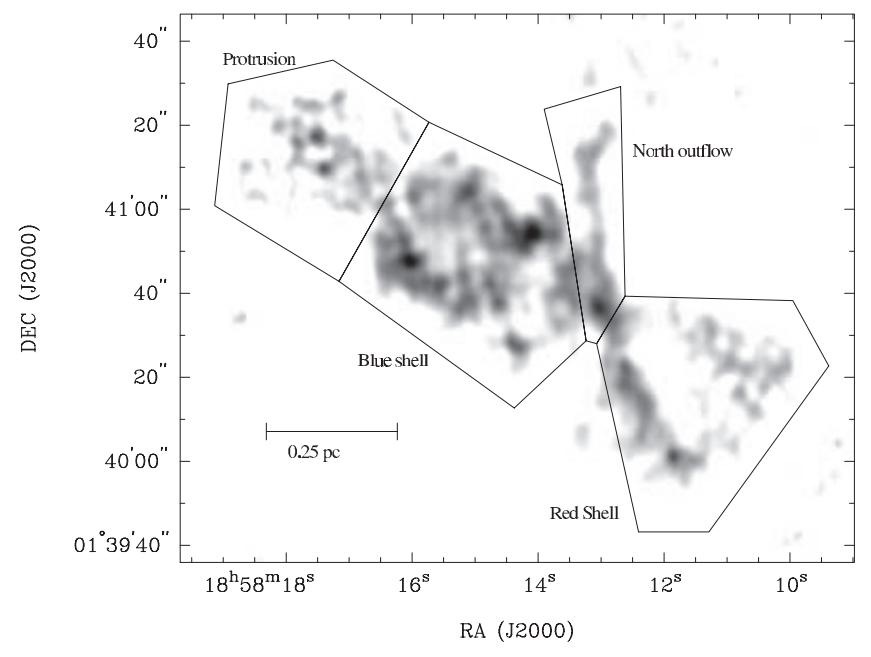

Fig. 1. The integrated ${ }^{12} \mathrm{CO} J=1 \rightarrow 0$ map with the boundaries used to define the differents features of the outflow overlayed and labelled. The synthesised beam is $3.4^{\prime \prime} \times 2.8^{\prime \prime}$ at a PA of $-12^{\circ}$.

angular resolution of these data has revealed more structure than was apparent in previous $\mathrm{CO}$ maps. In addition to the NE-SW structure, a N-S finger of $\mathrm{CO}$ emission appears to emanate from the central region (Fig. 1), a feature only formerly seen in the CO $J=3 \rightarrow 2$ (Gibb et al. 2003). This CO filament is coincident with the infrared and radio jet identified in this source. Also obvious in this data is that the main $\mathrm{CO}$ flow has a limb brightened appearence, suggesting the presence of a shell structure. However, when interpreting this structure, the spatial frequency filtering of the array need to be considered.

Nevertheless the velocity structure of the emission (Fig. 3) also appears consistent with an expanding shell, particularly for the NE lobe of the outflow. This evidence can be seen in Fig. 3, where the panels at $v=40,28$, and $8 \mathrm{~km} \mathrm{~s}^{-1}$, suggest the presence of a receding face of the NE shell, the laterally expanding limb, and the approaching NE shell material respectively. The structure of the SW lobe of the outflow is somewhat more confused, although it is also suggestive of a shell structure with the limbs of the shell apparent at a velocity of $\sim 40 \mathrm{~km} \mathrm{~s}^{-1}$. The approaching and receding faces of the shell can be seen at $36 \mathrm{~km} \mathrm{~s}^{-1}$ and $48 \mathrm{~km} \mathrm{~s}^{-1}$ respectively. Examination of the data suggests two factors which may be contributing the more confused nature of the SW lobe. First the emission appears more signficantly affected by the filtered emission close to the line core. But in addition a narrow component of redshifted $\mathrm{CO}$, which may trace the receding counterpart of north-south oriented blueshifted finger of emission, appears to overlap with the south eastern limb of the putative shell.

The structure of the ambient medium may also influence the structure of the outflow emission, contributing to the difference between the NE, blue, and SW, red, lobes as well as the clumpy nature of the emission. The red lobe is significantly less extended along the outflow axis, its emission covers less velocity than the blue lobe and its integrated intensity is lower. We have identified four spatially distinct regions in the outflow. (These can also be seen in Fig. 1.) The blue lobe has a spike of material (labelled as the protrusion in Fig. 1) beyond the main cavity which contains the most blue-shifted emission in the outflow. Spectra spatially integrated over each of these regions can be seen in Fig. 4. 

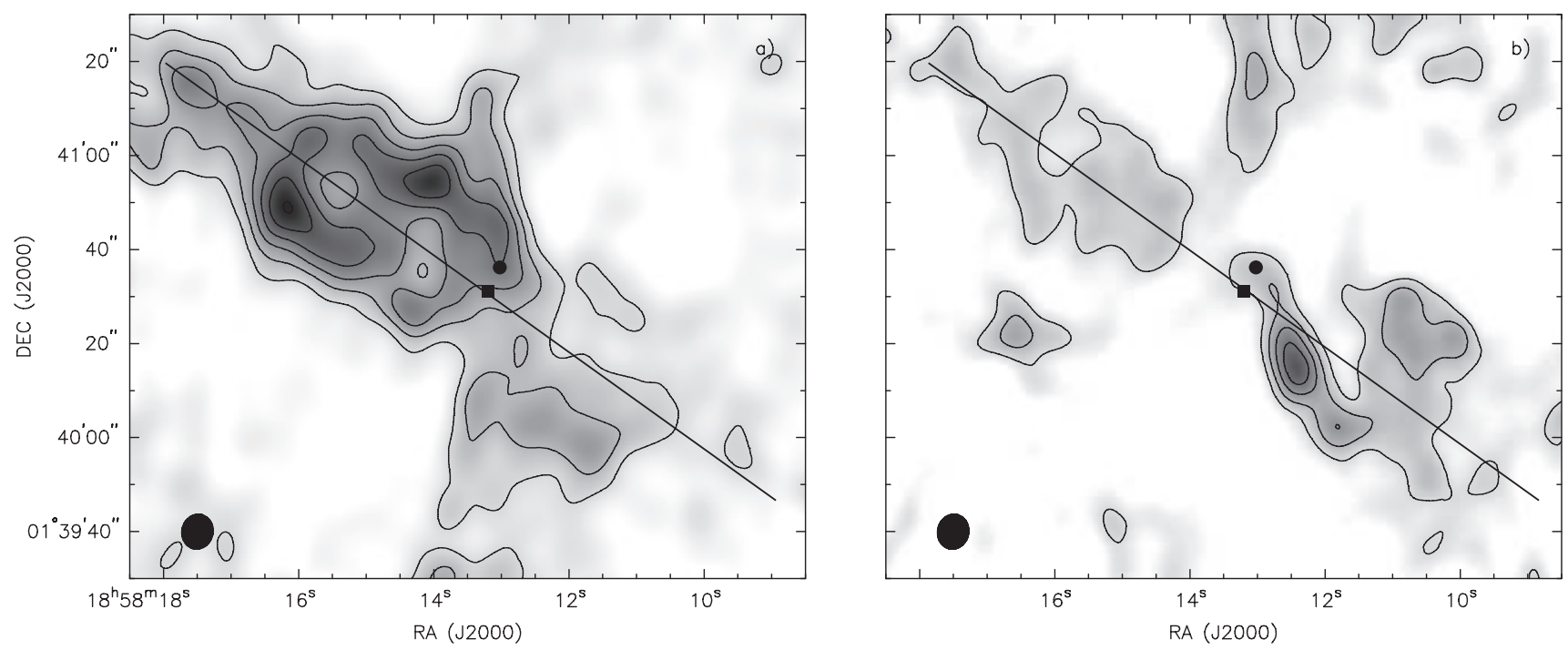

Fig. 2. CO $J=1 \rightarrow 0$ emission integrated between a) -10 and $30 \mathrm{~km} \mathrm{~s}^{-1}$ showing the blueshifted emission (left) and b) 38 and $60 \mathrm{~km} \mathrm{~s}$ showing the redshifted emission (right). The blueshifted emission is shown with contour levels at 0.4 to 2.4 in steps of 0.4 Jy per beam and has a noise level of $0.12 \mathrm{Jy}$ per beam. The redshifted emission is shown with contour levels at 0.5 to 2 in steps of $0.3 \mathrm{Jy}$ per beam and has a noise level of $0.14 \mathrm{Jy}$ per beam. The radio source G35.2N is marked with a circle and the millimetre source G35MM2 is marked with a square at an offset of (2.8, -5.2) arcsec. The circle in the bottom left of each map represents the beam FWHM. The UV data from which these images have been produced has been weighted to give a synthesised beam of $7.5^{\prime \prime} \times 6.9^{\prime \prime}$ (at a PA of $-21^{\circ}$ ) for clarity. The diagonal line has been overlaid to highlight the alignment of the expanding red and blue CO shells with the source G35MM2.

\subsection{Continuum}

Since the $2.7 \mathrm{~mm}$ continuum emission, presumably due to warm dust, was too weak to detect with the longer baselines, Fig. 5a was produced from the D array data only. The emission is not significantly resolved but peaks between the two sources, G35.2N and G35MM2. As the location of the peak is not particularly biased towards either source, we hypothesise that the two sources have a similar mass, contributing more or less equally to the unresolved emission.

The total mass of dust and gas calculated from the total dust emission was found using,

$M=g S_{v} d^{2} / \kappa_{v} B_{v}\left(T_{\text {dust }}\right)$

where $S_{v}$ is the flux density at frequency $v, d$ is the distance to the source, $\kappa_{v}$ is the absorption coefficient, $g$ the gas to dust ratio and $B_{v}\left(T_{\text {dust }}\right)$ is the Planck function at frequency $v$ for a blackbody of temperature $T_{\text {dust }}$. Assuming a dust temperature of $33 \mathrm{~K}$ for consistency with previous authors (i.e. Gibb et al. 2003) and extrapolating the values given by Ossenkopf \& Henning (1994) to give $\kappa_{2.7 \mathrm{~mm}}=0.03 \mathrm{~cm}^{-2} \mathrm{~g}^{-1}$, we drive a mass of $\sim 130 M_{\odot}$.

\section{3. $C^{17} \mathrm{O}$ emission}

Like the continuum data above, the $\mathrm{C}^{17} \mathrm{O} J=1 \rightarrow 0$ was also best imaged using just the $\mathrm{D}$ array data. Unlike the dust emission, the $\mathrm{C}^{17} \mathrm{O}$ clearly peaks close to G35MM2 (Fig. 5b) and appears resolved along the axis perpendicular to the outflow. The $\mathrm{C}^{17} \mathrm{O}$ occupies the same area as the disk-like structure known to be present (Dent et al. 1985; Gibb et al. 2003), however no velocity gradient is apparent in the along the major axis of the $\mathrm{C}^{17} \mathrm{O}$. We measure a 2:1 aspect ratio from the $\mathrm{C}^{17} \mathrm{O}$ contours and estimate a size of $20^{\prime \prime} \times 10^{\prime \prime}$ after correcting for the beam.

The mass calculated from the intensity of $\mathrm{C}^{17} \mathrm{O}$ followed the method outlined later in this paper for the ${ }^{12} \mathrm{CO} J=1 \rightarrow 0$ outflow emission. We assumed the $\mathrm{C}^{17} \mathrm{O}$ emission to be optically thin, $T_{\mathrm{ex}}=33 \mathrm{~K}$, a $\mathrm{C}^{17} \mathrm{O}$ abundance of $4 \times 10^{-7}$ and we integrate over all three hyperfine components (Ladd et al. 1998). We find a mass of $\sim 44 M_{\odot}$.

Fitting Gaussians to the hyperfine $\mathrm{C}^{17} \mathrm{O}$ spectrum (see Fig. 6, Ladd et al. 1998) gives $1.8 \pm 0.6 \mathrm{~km} \mathrm{~s}^{-1}$ for the FWHM of the velocity dispersion. Calculating the virial mass using $3 \sigma^{2}=$ $G M / R$, we find $M_{\text {virial }}=40 \pm 19 M_{\odot}$ which is in reasonable agreement with the mass from the $\mathrm{C}^{17} \mathrm{O}$ column density.

\section{Analysis of outflow parameters}

In calculating the outflow characteristics we adopt the approach outlined in Cabrit \& Bertout (1990). However, calculation of the effect of optical depth is hindered by the lack of observations of an optically thin isotopomer for the $J=1 \rightarrow 0$ transition e.g. ${ }^{13} \mathrm{CO} J=1 \rightarrow 0$. However the optical depth has been calculated using this method for the $J=3 \rightarrow 2$ transition by Gibb et al. (2003). They found the optical depth for $J=3 \rightarrow 2$ transition could be reasonably described by the relation $\tau(v) \simeq 57 / v^{1.7}$, where $v$ is the velocity in $\mathrm{km} \mathrm{s}^{-1}$. We can use this relation to estimate the optical depth for the $J=1 \rightarrow 0$ transition. Considering the transitions $J=3 \rightarrow 2$ and $J=2 \rightarrow 1$ we can write:

$\frac{\tau_{3-2}}{\tau_{2-1}}=\frac{3}{2}\left[\frac{1-\exp \left(-h v_{3-2} / k T_{\mathrm{ex}}\right)}{\exp \left(h v_{2-1} / k T_{\mathrm{ex}}\right)-1}\right]$.

Multiplying the similar formula for $J=2 \rightarrow 1$ to $J=1 \rightarrow 0$ ratio we obtain:

$\frac{\tau_{3-2}}{\tau_{1-0}}=\frac{3}{\exp \left(h v_{2-1} / k T_{\mathrm{ex}}\right)}\left[\frac{1-\exp \left(-h v_{3-2} / k T_{\mathrm{ex}}\right)}{\exp \left(h v_{1-0} / k T_{\mathrm{ex}}\right)-1}\right]$.

Using this we calculated the optical depth of the $J=1 \rightarrow 0$ emission as a function of frequency using the $J=3 \rightarrow 2$ result derived by Gibb et al. (2003).

The column density of $\mathrm{H}_{2}$ was calculated on a channel-bychannel basis using,

$N_{\text {chan }}=\left(\frac{\mathrm{H}_{2}}{{ }^{12} \mathrm{CO}}\right) \times \frac{3 k T_{\mathrm{ex}}}{8 \pi^{3} \mu^{2} v} \times \frac{\left(1-\mathrm{e}^{h v / k T_{k}}\right)^{-1}}{J_{v}\left(T_{k}\right)-J_{v}\left(T_{\mathrm{b}}\right)} T_{\mathrm{A}}(v) \Delta v$ 


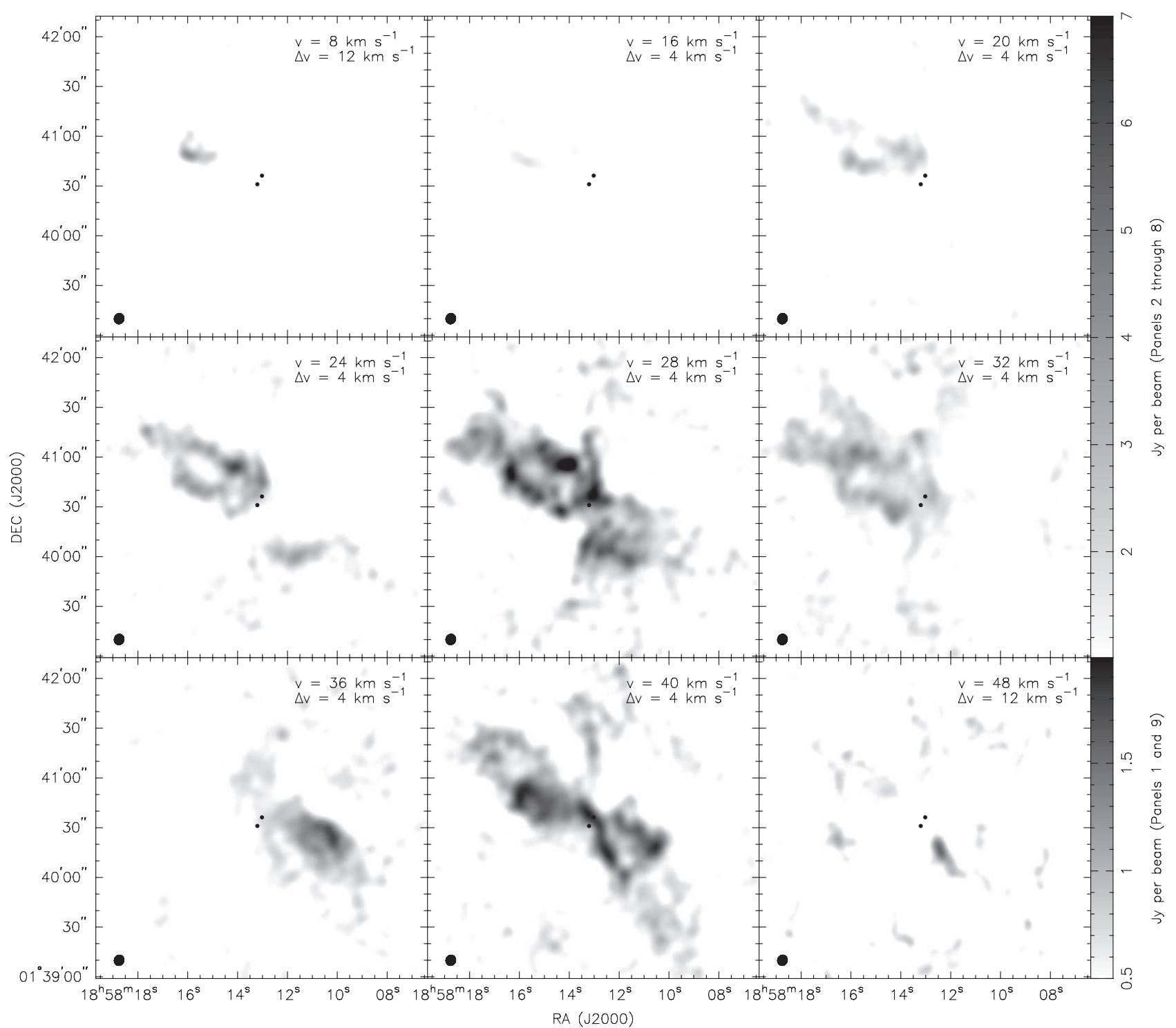

Fig. 3. ${ }^{12} \mathrm{CO} J=1 \rightarrow 0$ emission integrated over 12 or $4 \mathrm{~km} \mathrm{~s}^{-1}$ wide intervals between 2 and $54 \mathrm{~km} \mathrm{~s}^{-1}$. The two extreme velocity intervals have been integrated over $12 \mathrm{~km} \mathrm{~s}^{-1}$, and all other intervals over $4 \mathrm{~km} \mathrm{~s}^{-1}$. The images where produced using a weighting set by robust $=0.25$ and the clean beam is represented by the filled circle in the lower left of each panel. The positions of G35.2N and G35MM2 are marked by the two symbols near the map centre, G35.2N being the most northern. The scalebar on the bottom right corresponds to the top left and lower right panels (showing the emission centred at $8 \mathrm{~km} \mathrm{~s}^{-1}$ and $48 \mathrm{~km} \mathrm{~s}^{-1}$ ), the upper scalebar corresponds to the remaining 7 plots. The synthesised beam is $6.4^{\prime \prime} \times 5.9^{\prime \prime}$ at a PA of $-22^{\circ}$.

and then corrected for optical depth by a factor of $\tau /\left(1-\mathrm{e}^{-\tau}\right)$. We adopted a ${ }^{12} \mathrm{CO}$ abundance to be $10^{-4}$ and assumed $T_{\mathrm{ex}}=$ $T_{k}=33 \mathrm{~K}$. Summing over all than channels for each part of the outflow, the mean velocity of the outflowing material was calculated by dividing the momentum by the mass; the width adopted was the size of the shell perpendicular to the outflow axis and the age is calculated by considering the material to have been expanding from the source of the outflow at the observed velocity of the shell front.

The data has been analysed for the four spatially distinct features in the CO map and shown in Fig. 1: the blue shell; the red shell; the north pointing $\mathrm{CO}$ outflow; the $\mathrm{CO}$ protrusion beyond the main blue shell. Table 1 lists the results for each region.

The optical depth derived for the $J=1 \rightarrow 0$ emission ranges from 0.01 to 1.1 for the range of velocities covered by the outflow. This range leads to optical depth corrections to the derived mases in the range of 1.66 and 1 . The total mass estimate changes by a factor of 1.23 when accounting for optical depth; the other parameters change by corresponding amounts.

\subsection{The mass-velocity relation}

A similar method was employed to calculate the mass of the outflow on a channel by channel basis and apply it to the four regions of the outflow separately to obtain the mass as a function of velocity relation for each feature of the outflow. Figure 7 shows the mass as a function of velocity relation for the red- and blue-shifted gas for each region. The mass was first calculated for each channel in the data (the channel width is $1.02 \mathrm{~km} \mathrm{~s}^{-1}$ ). The results shown by as open triangle symbols. At high velocities the mass per channel data becomes rather scattered. We can reduce the noise by reprocessing the data at a larger channel width, which produces superior results to simply re-binning the data, as the deconvolution will be more accurate given a better 


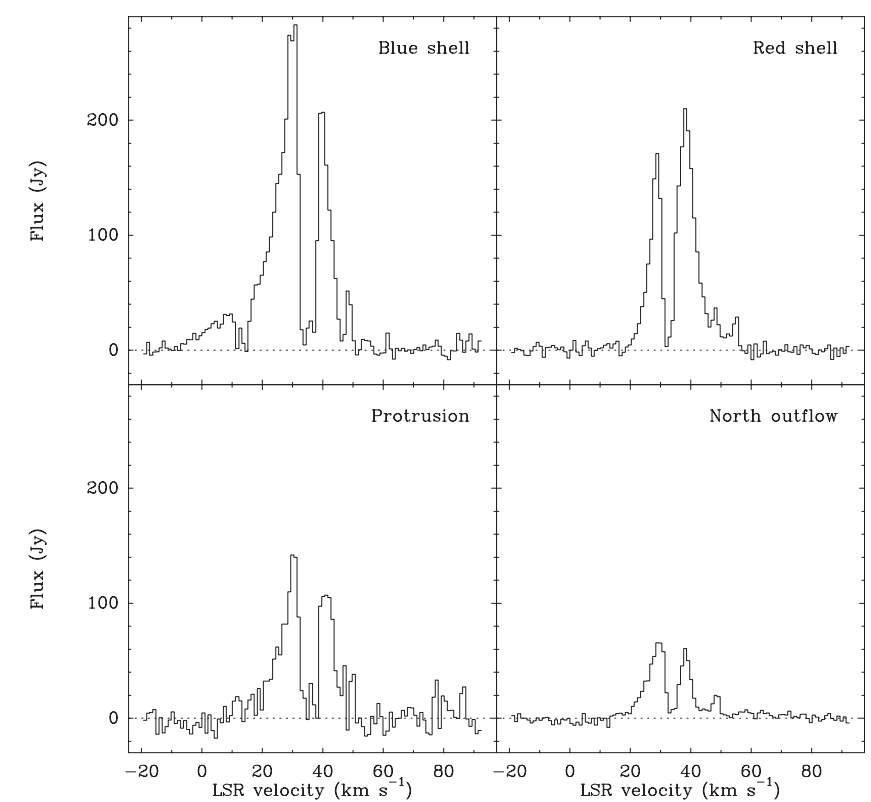

Fig. 4. ${ }^{12} \mathrm{CO} J=1 \rightarrow 0$ spectra obtained by integrating over each region as shown in Fig. 1. The amount of noise varies due to the number of overlapping pointings in the mosaic as well as the area of the region.
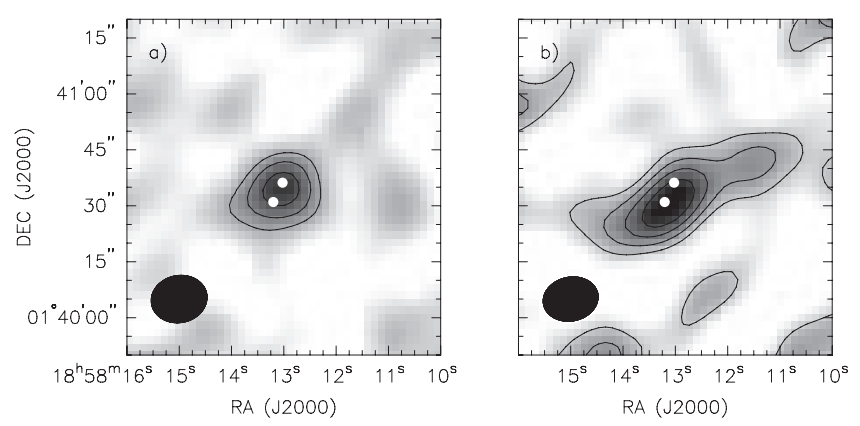

Fig. 5. a) The $2.7 \mathrm{~mm}$ continuum emission. The contours are set at 0.3 , 0.35 and $0.4 \mathrm{Jy}$ per beam. The noise level is $0.05 \mathrm{Jy}$ per beam. The synthesised beam is $16.1^{\prime \prime} \times 12.7^{\prime \prime}$ at a PA of $-82^{\circ}$. b) The $\mathrm{C}^{17} \mathrm{O} J=$ $1 \rightarrow 0$ emission integrated over the entire velocity range. The noise level is $0.11 \mathrm{Jy}$ per beam. The synthesised beam is $16.8^{\prime \prime} \times 12.7^{\prime \prime}$ at a PA of $-81^{\circ}$. The contours are set at $1,1.08,1.16$ and 1.24 Jy per beam. Both maps show the location of G35.2N, G35MM2 and the shape of the beam in the lower left.

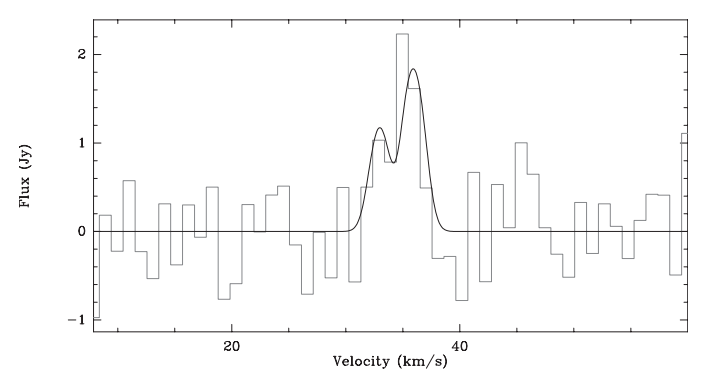

Fig. 6. $\mathrm{C}^{17} \mathrm{O} J=1 \rightarrow 0$ spectrum overlaid with a Gaussian fit to the hyperfine components.

defined image. The data was reprocessed in $5 \mathrm{~km} \mathrm{~s}^{-1}$ channels and results of a recalculation of the mass is shown with filled squares in the plots. The good agreement between the two sets of data shows the data reduction is working well. Power laws of the form $v^{-\gamma}$ have been fitted where appropriate and the results shown in Table 2 and plotted in Fig. 7. The mass of the
Table 1. Outflow parameters corrected for optical depth.

\begin{tabular}{lll}
\hline \hline Parameters & Blue shell & Red shell \\
\hline Mass $\left(M_{\odot}\right)$ & 5.45 & 4.27 \\
Momentum $\left(M_{\odot} \mathrm{km} \mathrm{s}^{-1}\right)$ & 45.2 & 27.7 \\
Energy $(\mathrm{erg})$ & $11.9 \times 10^{45}$ & $4.67 \times 10^{45}$ \\
Mean velocity $\left(\mathrm{km} \mathrm{s}^{-1}\right)$ & 8.29 & 6.48 \\
Size $(\mathrm{pc})$ & 0.29 & 0.24 \\
Dynamic Age $(\mathrm{yr})$ & 33300 & 35600 \\
\hline & North outflow & Protrusion \\
\hline Mass $\left(M_{\odot}\right)$ & 0.8 & 2.66 \\
Momentum $\left(M_{\odot} \mathrm{km} \mathrm{s}^{-1}\right)$ & 5.94 & 22.22 \\
Energy $(\mathrm{erg})$ & $1.04 \times 10^{45}$ & $6.27 \times 10^{45}$ \\
Mean velocity $\left(\mathrm{km} \mathrm{s}^{-1}\right)$ & 7.43 & 8.35 \\
\hline
\end{tabular}

Table 2. Power fit index $\gamma$ for the red- and blue-shifted gas in each region for each velocity range.

\begin{tabular}{lll}
\hline \hline Region & Vel. range $\left(\mathrm{km} \mathrm{s}^{-1}\right)$ & $\gamma$ \\
\hline NE shell blue & $5-10$ & $1.5 \pm 0.2$ \\
& $10-35$ & $1.7 \pm 0.1$ \\
NE shell red & $35-45$ & $5.6 \pm 0.3$ \\
SW shell blue & $6-10$ & $2.2 \pm 0.05$ \\
& $10-15$ & $3.0 \pm 0.2$ \\
NS shell red & $6-20$ & $5.5 \pm 0.1$ \\
N outflow blue & $5-10$ & $2.5 \pm 0.2$ \\
& $10-13$ & $1.7 \pm 0.2$ \\
Protrusion blue & $4-13$ & $3.1 \pm 0.2$ \\
\hline
\end{tabular}

outflow gas is dominated by the material moving with the lowest velocities.

\section{Modelling}

For the purpose of studying the physics in this outflow we have created a simple 2D hydrodynamic model following an approach similar to that of Lee et al. (2001) for low-mass sources. The model makes use of the ZEUS-2D hydrodynamic code (Stone \& Norman 1992) to solve the equations of hydrodynamics,

$\frac{D \rho}{D t}+\rho \nabla \cdot v=0$

$\rho \frac{D v}{D t}-\nabla p=0$

$\rho \frac{D}{D t}\left(\frac{e}{\rho}\right)=-p \nabla \cdot v-n^{2} \Lambda$

where $\rho, n, v, p$, and $e$ are the mass density, the H number density, velocity, thermal pressure and energy density respectively. Gravity is ignored in these simulations. The code has been modified to include the cooling function, $\Lambda$, from Rosen $\&$ Smith (2003), which includes a simple chemistry to calculate the abundance of the cooling molecular species and advection code for the fraction of hydrogen in molecular versus atomic form.

The parameters of the model have been chosen to replicate the conditions in G35.2N. It is likely that the exact structure of the wind from a young star is affected the density structure in the material around the driving source, however for simplicity we assume the wind flows into a uniform density medium. In any case there are few constraints on the density structure in the region into which the G35.2N outflow is flowing. We have estimated the ambient density by assuming the mass calculated for 


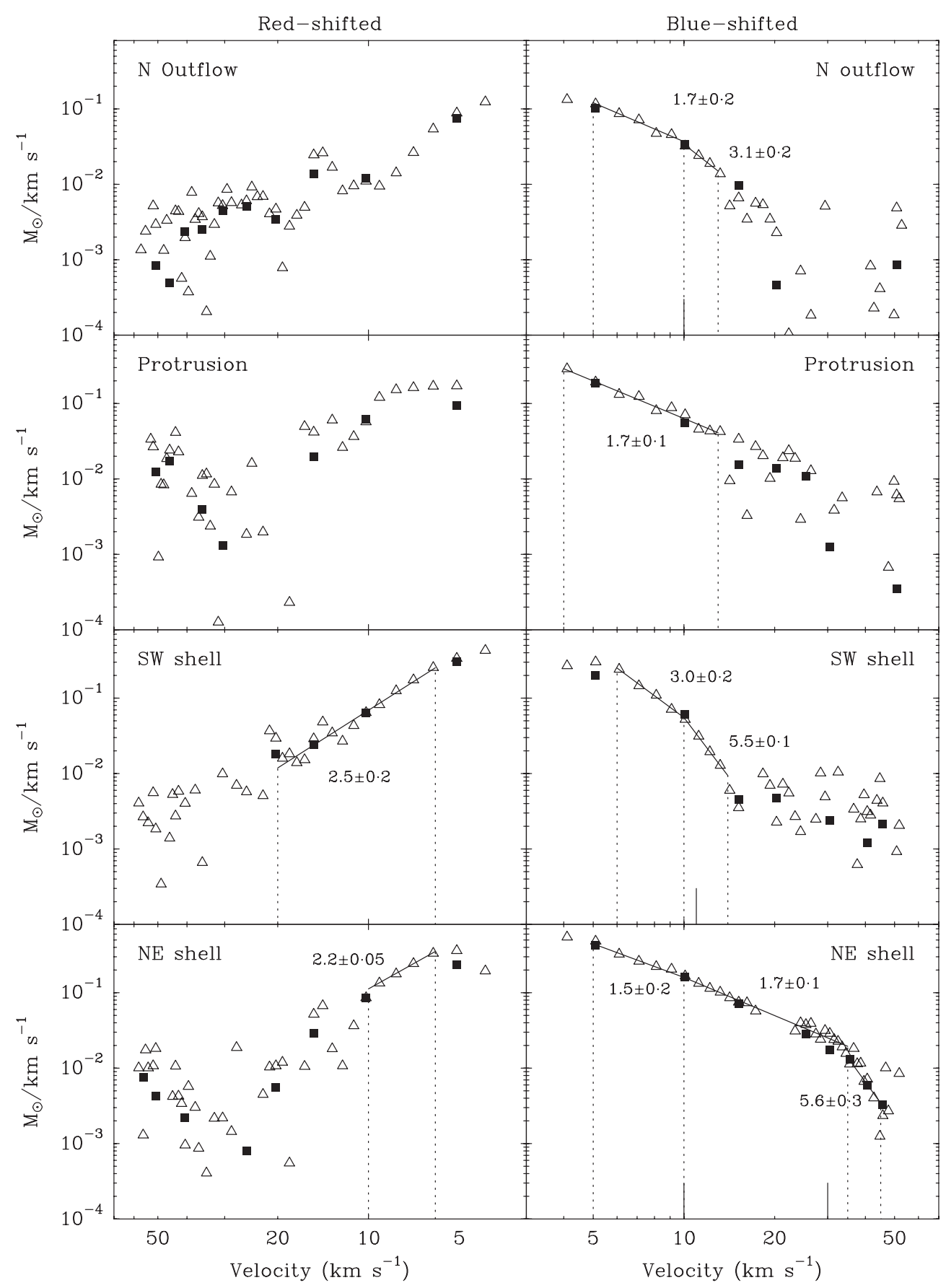

Fig. 7. Mass-velocity plots for the redshifted and blue-shifted gas from each region of the outflow (see Fig. 1). Power laws have been fitted where appropriate. The plots show the mass distribution calculated from $1.02 \mathrm{~km} \mathrm{~s}^{-1}$ channels with open triangles and mass distribution calculated from $5 \mathrm{~km} \mathrm{~s}^{-1}$ channels with filled squares. The velocity of the relavent breaks in power law seen in the CO $J=3 \rightarrow 2$ (Gibb et al. 2003) are marked with a short solid vertical line at the bottom of each plot.

the shells of the outflow has been swept up from the volume they occupy, this gives $\rho=1.67 \times 10^{-20} \mathrm{~g} \mathrm{~cm}^{-3}\left(n=4 \times 10^{3} \mathrm{~cm}^{-3}\right)$ for the blue shell: a reasonable value which supports the main outflow having a shell structure. We assume the temperature of the ambient medium to be $30 \mathrm{~K}$. The kinematic age of the outflow of 30000 years is used as the end time for all model runs.

The equations are solved on a regular 2D grid in sphericalpolar coordinates. The number of zones in the grid is set to $600 \times$ 400 which translates to a domain of $(R, \theta)=\left(8 \times 10^{16}-4 \times\right.$ $\left.10^{18} \mathrm{~cm}, 0-\pi / 2\right)$ with a spatial resolution of $6.53 \times 10^{15} \mathrm{~cm}$ in $r$ and $3.93 \times 10^{-3}$ radians in $\theta$. (Models were also run with twice this resolution in each coordinate. These models showed no evidence of the resolution of the simulations affecting the results.) We introduce an atomic wind by setting the boundary conditions along the inner $r$ boundary to inflow. The wind takes the form derived by Shu et al. (1995), with a density $\rho_{\mathrm{w}} \propto$ $1 /(r \sin \theta)^{2}$. Matzner \& McKee (1999) show that for a general hydromagnetic wind the velocity of the wind is constant across the streamlines at large distances from the source, it seems plausible to assume that the wind velocity, $v_{\mathrm{w}}$, does not vary too widely and so we assume it is constant with respect to $\theta$ with a value of $30 \mathrm{~km} \mathrm{~s}^{-1}$. Within an angle $\theta=0.01$ radians, density, $\rho_{j}$, is assumed constant (Tan \& McKee 2002), the value of which is used to vary the jet component of the wind. Reflecting 


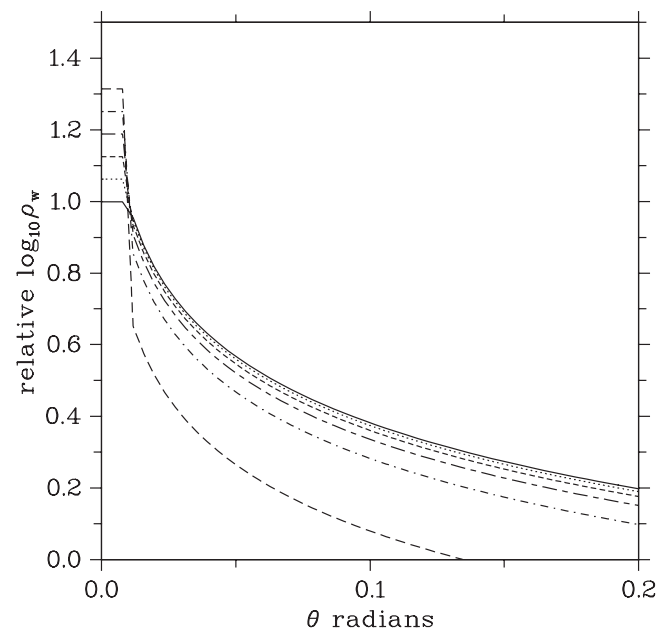

Fig. 8. The graph shows the six wind profiles used in the Jet and wind comparison models. Only the density for small $\theta$ is plotted to show how the momentum in the jet-like component and the hydromagnetic wind are distributed. The density shown is relative to the density of the lowest density jet-like component.

boundary conditions are used for the inner and outer $\theta$ boundaries, and an outflow boundary condition is used for the outer $\mathrm{R}$ boundary.

\subsection{Jet and wind comparison}

Comparisons of the model outflows resulting from using jetdriven versus wide-angled-winds can help determine the physical processes behind the formation of a molecular outflow. This is of particular interest in massive star formation as observations of jets with associated outflows are relatively rare. As we have parameterised our wind profile in terms of components that can be related to a jet and wide-angled-wind, we can attempt to determine whether one or a combination of both of these components best reproduces our observations of G35.2 massive molecular outflow.

We varied the jet component of the wind by redistributing the momentum between the $\theta<0.01$ and $\theta>0.01$ domains of the wind (see Fig. 8). We investigated models where the distribution of momentum was varied so that between $9 \%$ and $92 \%$ was contained in the central jet-like component (the jet). The choice of $\sim 92 \%$ of the momentum flux distributed at $\theta>0.01$ produces a smooth wind density profile: the constant density of the jet-like component, within the region $\theta<0.01$, component is equal to the density of the general hydromagnetic wind at $\theta=0.01$. The results of these models are shown in Fig. 9.

As the NE lobe of the observed outflow has the strongest emission and appears less confused we concentrate our model comparisons with this region of the outflow anyway. Comparing these results to our observational data, we see that the NE lobe of the outflow is well described by these models: consisting of a hollow shell close to the source and a narrow tip toward the end of the outflow. As the fraction of the momentum in the jet-like component of the wind is increased the resulting outflow lobe appears increasingly more elongated and collimated. The "pure" hydromagnetic wind model, the model with the least jet-like component, qualitatively produces the best match to the shape of NE outflow lobe for this range of model parameters.
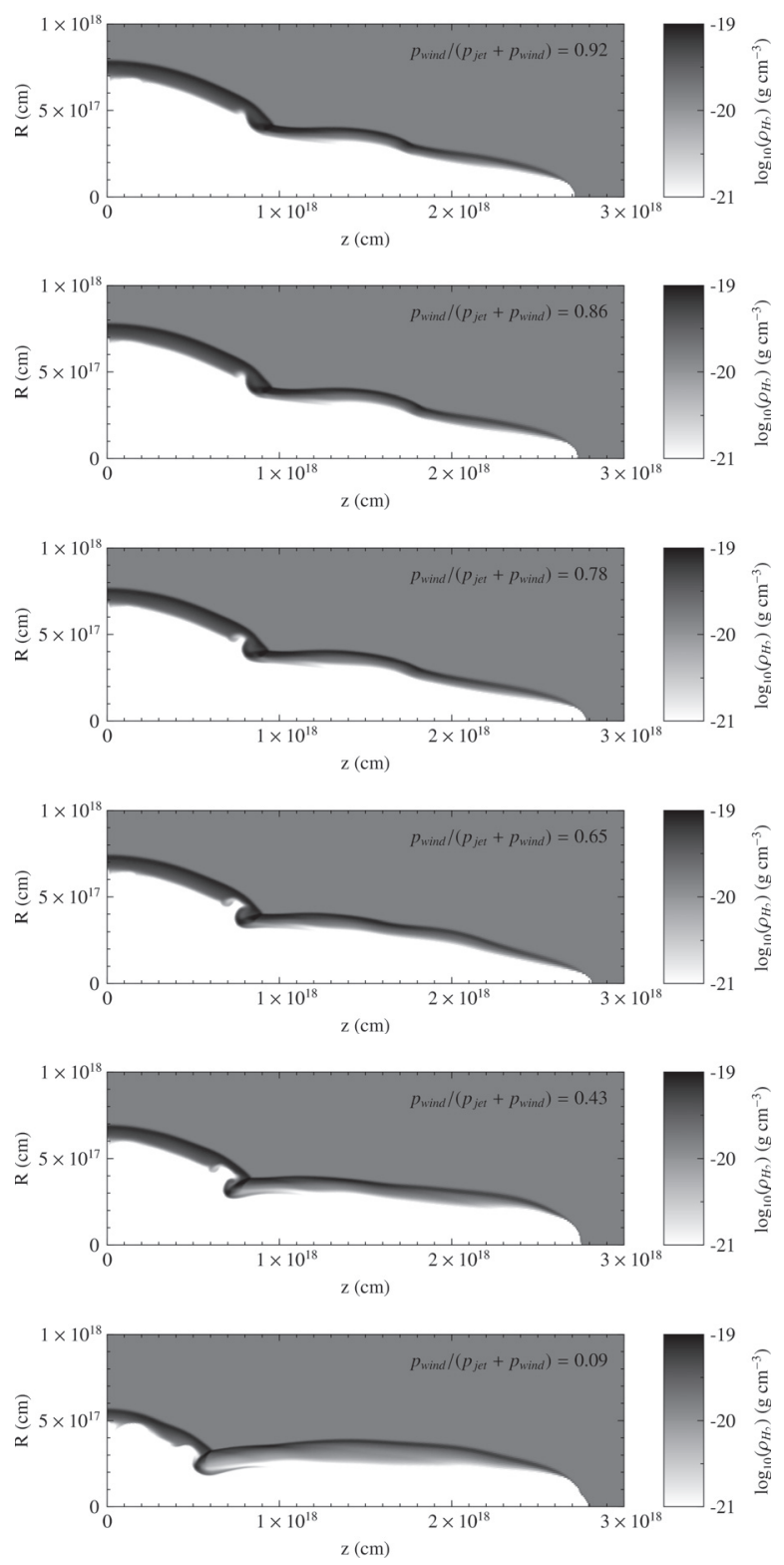

Fig. 9. The above six plots show the density of $\mathrm{H}_{2}$ after 30000 years of evolution with a constant wind profile over the duration of each model. From top to bottom the models have an increasing fraction of the total momentum in the jet, which has momemtum $p_{\text {jet }}$ compared to the hydromagnetic wind, which has total momentum $p_{\text {wind }}$. The top plot shows a model with $92 \%$ of total the wind momentum introduced via the general hydromagnetic wind and the remaining $9 \%$ in the jet-like component $(\theta<0.01$ radians). The bottom plot shows the result if $92 \%$ of the momentum is introduced via the jet-like component of the wind. Figure 8 shows the six different profiles used. Qualitatively the observations appear most similar in structure to the top most panel corrsponding to an input wind which does not have an enhanced on-axis, jet-like, component.

\subsection{Wind evolution}

Another scenario one could imagine for the wind from a massive young stars is the evolution of the distribution of the wind as the star forms. This is might be appropriate for models in which mass accretion forming the central stars also varies in time (e.g. Tan \& McKee 2002).

Figure 10 shows two simulations with time varying wind profiles. These two models compare the resulting outflow for 

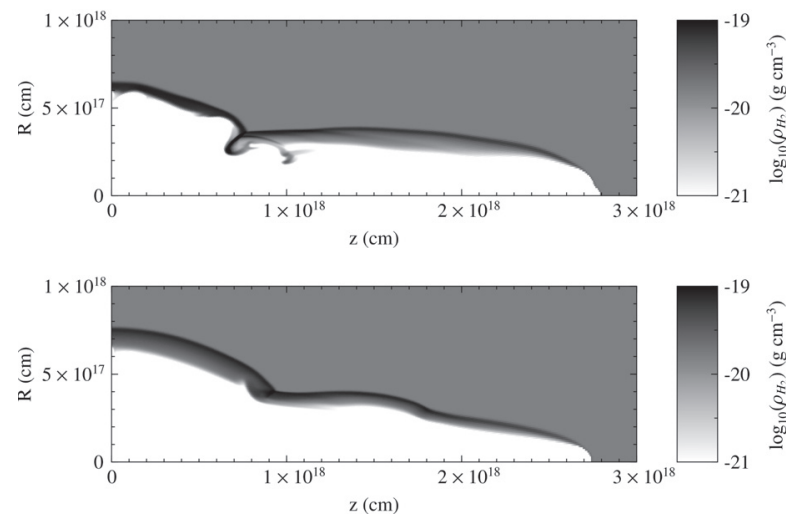

Fig. 10. The upper plot initially has $92 \%$ of the momentum flux in $\theta<0.01$ radians. The momentum flux linearly changes with time, so after 30000 years $91 \%$ of the momentum flux is distributed at $\theta>0.01$ radians. The lower plot shows the result if $91 \%$ of the momentum flux begins in $\theta>0.01$ radians and evolves in the opposite sense.

a wind initially having $\sim 92 \%$ of the momentum being injected within $\theta<0.01$ and by the end of the simulation having $~ 92 \%$ of the momentum at $\theta>0.01$ radians, and then a model evolving in the opposite sense. Comparing these results with those in Fig. 9 if can be seen that the resulting shape of the outflow lobe is predominately influenced by the initial wind conditions, rather than its later evolution.

\subsection{Total momentum input}

In Fig. 11 we show the results of investigating changing the wind momentum flux, first doubling, and then quadrupling the initial rate of momentum input used in the previous models. The models show that increasing the total rate of momentum input (whilst keeping the wind velocity $v_{\mathrm{w}}$ the same) increases the distance the off-axis wind driven shell propagates more than the already over-dense jet-like $(\theta<0.01)$ component, producing less elongated outflow lobes. The explanation of this effect is that the $\theta<0.01$ wind component is sufficiently dense not to be slowed significantly by the ambient medium, so increasing its density further will do little to increase the distance it propagates within the fixed time-span of the model.

The effect of this scaling the momentum flux has on the shape of the outflow could, perhaps, explain the difference in collimation and shape seen between high and low-mass outflows. For the particular case of G35.2N, increasing the total momentum flux of the wind produces a closer match to the shape of the outflow lobe.

\section{Discussion}

G35.2-0.74N is a rare example of a massive young star associated with a jet and a $\mathrm{CO}$ ouflow. The data clearly do show a weak filament of $\mathrm{CO}$ emission associated with the jet, but the overall impression is that the jet and dominant NE-SW CO outflow in this region are separate entities. If we assume that the $\mathrm{CO}$ emission close to G35.2-0.74N is not solely part of the main NE-SW outflow, then the location and shape of the NE and SW shells and the protrusion implies the centre of the main outflow lies closer to G35MM2 than G35.2N, suggesting G35MM2 as the driving source of the major outflow.

Although the resolution of our continuum and $\mathrm{C}^{17} \mathrm{O}$ images do not allow us to fully resolve the individual contributions from
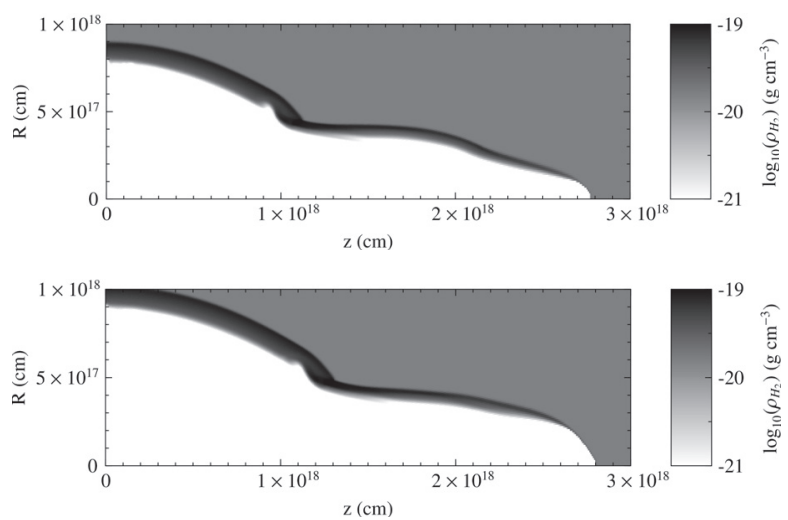

Fig. 11. Both plots show the results of models run with the wind profile used in the model shown first in Fig. 9, except the total momentum input is double (upper plot) and quadruple (lower plot) the quantity previously used. Notice the decrease in the apparent collimation as the total momentum is increased.

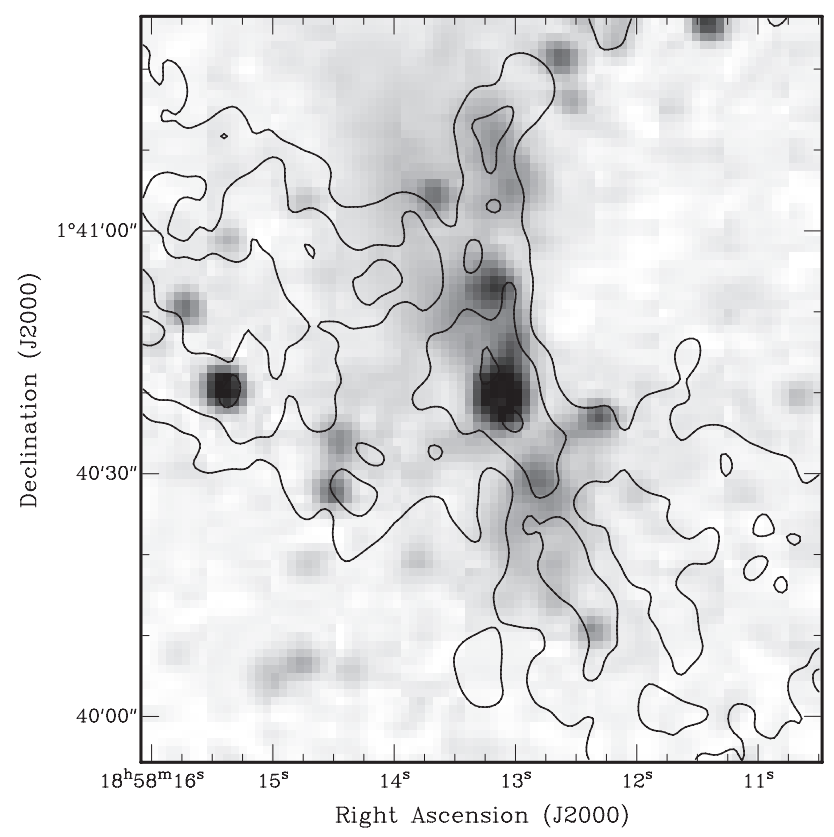

Fig. 12. $K(2.2 \mu \mathrm{m})$ image of the region from the 2 Mass survey showing the reflection nebulae associated with the northward orientated outflow.

G35MM2 and G35.2N, the differences between the images in these two tracers suggests different distrbutions of material. The continuum suggests the presence of similar mass of dust around each source, whereas the $\mathrm{C}^{17} \mathrm{O}$ data clearly peaks on G35MM2 and not G35.2N, suggesting that there is more gas associated with the G35MM2 source. The presence of so much gas towards G35MM2 would appear to suggest that it is the younger source, yet it also appears to be the driving source of the larger outflow.

If indeed $\mathrm{G} 35.2 \mathrm{~N}$ is the somewhat older source, having dispersed is circumstellar gas, then the northward pointing CO filament and the radio/infrared jet associated with it are tracing a more evolved outflow. The $K(2.2 \mu \mathrm{m})$ image (Fig. 12) of this region appears to support this interpretation. The extended, 
nebulous $K$ emission traces a large region coincident with the blueshifted CO filament suggesting it is light scattered off the inner cavity shell created by the older northward pointing outflow. However the short recombination timescale ( 100 years) for the ionised component of the jet indicates that it mostly likely still being excited. There is little, if any $K$ emission associated with the main $\mathrm{CO}$ outflow from G35MM2, suggesting that even the outflow lobes are still heavily extinguished pointing to the youth of the system.

Counter-arguments may also be made to suggest that G35MM2 is older than G35.2N on account of its larger, less jet-like and more massive flow. Indeed the shell-like appearance and increased collimation with distance is reminiscent of the flow from the relatively evolved (although somewhat more luminous) source DR 21 (Garden et al. 1991; Davis \& Smith 1996). How, then, can we explain the tendency for molecular line emission to peak toward G35MM2 rather than G35.2N? The younger source would be expected to be more deeply embedded. A possible explanation is that the cooler gas around G35MM2 (being less luminous than $\mathrm{G} 35.2 \mathrm{~N}$ ) results in stronger emission in the low- $J$ lines of typical tracers, and that observations of higherexcitation lines would show a shift in the molecular peak toward G35.2N. Support for this interpretation comes from the ammonia $(2,2)$ observations of Little et al. (1985), which peak toward G35.2N. However, the ammonia $(3,3)$ observations of Brebner et al. (1987) clearly show the emission peaks at the position of G35MM2. Therefore, at the moment, it is not possible to distinguish one hypothesis unambiguously from the other and we propose further observations should be made using higherexcitation transitions.

The total mass of the outflowing $\mathrm{CO}$ is $13 M_{\odot}$, similar to the suggested mass of the central source, but only a small fraction of the total circumstellar mass of 40-140 $M_{\odot}$ implied by the continuum and $\mathrm{C}^{17} \mathrm{O}$ emission. The mass of the outflow is reasonable agreement with previous estimates for this outflow. Dent et al. (1985) found a mass of $30 M_{\odot}$ from single dish observations of the same $J=1 \rightarrow 0$ transitions while Gibb et al. (2003) deduced a mass of $11 M_{\odot}$ from observations of the $J=3 \rightarrow 2$ transition. Compared to other young systems of similar total luminosity, $\sim 10^{4} L_{\odot}$, this is a typical outflow mass (Churchwell 1997; Beuther et al. 2002).

\subsection{The mass-velocity relation}

The mass-velocity relation of outflows can often be described by two power laws with the break at $v=10-30 \mathrm{~km} \mathrm{~s}^{-1}$ (see references within Downes \& Cabrit 2003). This feature is potentially useful as a constraint on outflow models. However, a recent study by Downes \& Cabrit (2003) has shown that the break in power law (seen first in the intensity-velocity relation) can be explained by molecular dissociation and the dependence of the emissivity on temperature: the emissivity initially increasing with $T$ up to a temperature similar to the excitation energy of the upper level of the transition, and at higher temperatures decreasing as $T^{-1}$. Hence, the underlying mass distribution can be described by a single power law while the intensity-velocity relation can exhibit a break.

Comparing the results of the analysis presented here for the ${ }^{12} \mathrm{CO} J=1 \rightarrow 0$ transitions with those of Gibb et al. (2003) for ${ }^{12} \mathrm{CO} J=3 \rightarrow 2$ we can see that many of the break points occur at the same velocity in both transitions. In particular the change in power law at approximately $10 \mathrm{~km} \mathrm{~s}^{-1}$ in the blue emission in the NE and SW outflow lobes, and interestingly in the north $\mathrm{CO}$ outflow also. The recurrence of this feature in a lower energy transition indicates that the temperature dependence of emissivity is not responsible for at least some of the breaks seen in the mass- or intensity-velocity plots. However, molecular dissociation would affect all transitions in the same manner. Indeed, direct comparison of the ${ }^{12} \mathrm{CO} J=3 \rightarrow 2$ and ${ }^{12} \mathrm{CO} J=1 \rightarrow 0$ line wings indicates the higher velocity material is warmer - consistent with dissociation occurring above a lower velocity limit.

\section{Conclusions}

High resolution observations of $\mathrm{CO} J=1 \rightarrow 0$ show the outflow in this region to be dominated by a $13 M_{\odot}$ outflow with its axis in a NE-SW direction. This outflow has a shell-like structure and we suggest that it is driven by the source G35MM2. In addition to the geometry of the outflow, this interpretation is supported by the detection of a separate component of $\mathrm{CO}$ emission coincident with the infrared and radio jet from the source G35.2N. The high mass of material detected in both the $2.7 \mathrm{~mm}$ continuum and $\mathrm{C}^{17} \mathrm{O}$ close to both the G35.2N and G35MM2 sources appear to confirm their high mass nature.

The CO filament and the radio and IR jet are associated with an extensive, bright $2.2 \mu \mathrm{m}$ nebula which is highly polarized (Walther et al. 1990) indicating that the emission is predominately radiation scattered from the edge of a cavity cleared by an outflow from the G35.2N source. On the other hand there is no such emission associated with the G35MM2 outflow, suggesting that the G35.2N outflow has cleared a larger region and is more evolved. The association of a radio/IR jet with what appears to be the older source suggests that the paucity of jets known to be associated with young high mass sources is not the result of jets being short lived phenomena early in the evolution of such objects.

By comparing the mass-velocity plots calculated from the ${ }^{12} \mathrm{CO} J=1 \rightarrow 0$ data with those of ${ }^{12} \mathrm{CO} J=3 \rightarrow 2$ (Gibb et al. 2003), we find common features in the power law representation of the relation. In particular, there are a number of breaks in the power law presentations that occur at the same velocity in the two transitions. If these breaks were a result of the temperature dependence of the emissivity of a particular molecular line transition, as suggested by Downes \& Cabrit (2003), then they should not occur at the same velocity in different transitions of CO. The existence of common break points suggests that molecular dissociation is is a better candidate for explaining at least some of the breaks in power law that are seen.

Our hydrodynamic simulations of this outflow show that a general hydromagnetic wind, without any additional jet component, can successfully produce the form of the massive molecular outflow from G35MM2 though it is difficult to conclusively rule out the presence of a jet. We also see that varying the wind profile over the duration of the model does not necessarily change the outflow shape from what the initial distribution of the wind would have determined. This suggests that the presence of time varying wind profiles might are hard to identify from the nature of the $\mathrm{CO}$ outflows they drive.

Lastly, we find that we were able to influence the shape and collimation of the resulting outflow by scaling the density of the wind whilst using the same overall form for the wind (and the same velocity). This may go some way to explain the apparent differences in shape between outflows from low- and high-mass objects.

Acknowledgements. J.R.B. wishes to acknowledge the Particle Physics and Astronomy Research Council (PPARC) for financially supporting the $\mathrm{PhD}$ studentship in which this work was completed. Research with BIMA is funded by 
grant AST-002893 from the National Science Foundation to the university of Maryland.

\section{References}

Beuther, H., Schilke, P., Sridharan, T. K., et al. 2002, A\&A, 383, 892 Brebner, G. C., Cohen, R. J., Heaton, B., \& Davies, S. R. 1987, MNRAS, 229, 679

Cabrit, S., \& Bertout, C. 1990, ApJ, 348, 530

Churchwell, E. 1997, ApJ, 479, L59

Davis, C. J., \& Smith, M. D. 1996, A\&A, 310, 961

Dent, W. R. F., Little, L. T., Kaifu, N., Ohishi, M., \& Suzuki, S. 1985, A\&A, 146,375

Downes, T. P., \& Cabrit, S. 2003, A\&A, 403, 135

Fuller, G. A., Zijlstra, A. A., \& Williams, S. J. 2001, ApJ, 555, L125

Garay, G., \& Lizano, S. 1999, PASP, 111, 1049

Garden, R. P., Geballe, T. R., Gatley, I., \& Nadeau, D. 1991, ApJ, 366, 474

Gibb, A. G., Hoare, M. G., Little, L. T., \& Wright, M. C. H. 2003, MNRAS, 339 , 1011
Heaton, B. D., \& Little, L. T. 1988, A\&A, 195, 193

Königl, A., \& Pudritz, R. E. 2000, Protostars and Planets IV, 759

Ladd, E. F., Fuller, G. A., \& Deane, J. R. 1998, ApJ, 495, 871

Lee, C., Stone, J. M., Ostriker, E. C., \& Mundy, L. G. 2001, ApJ, 557, 429

Little, L. T., Brown, A. T., Riley, P. W., et al. 1983, MNRAS, 203, 409

Little, L. T., Dent, W. R. F., Heaton, B., Davies, S. R., \& White, G. J. 1985 MNRAS, 217, 227

Little, L. T., Kelly, M. L., \& Murphy, B. T. 1998, MNRAS, 294, 105

Matzner, C. D., \& McKee, C. F. 1999, ApJ, 526, L109

Ossenkopf, V., \& Henning, T. 1994, A\&A, 291, 943

Richer, J. S., Shepherd, D. S., Cabrit, S., Bachiller, R., \& Churchwell, E. 2000, Protostars and Planets IV, 867

Rosen, A., \& Smith, M. D. 2003, MNRAS, 343, 181

Shu, F. H., Najita, J., Ostriker, E. C., \& Shang, H. 1995, ApJ, 455, L155+

Stone, J. M., \& Norman, M. L. 1992, ApJS, 80, 753

Tan, J. C., \& McKee, C. F. 2002, in Hot Star Workshop III: The Earliest Phases of Massive Star Birth, ASP Conf. Ser., 267, 267

Walther, D. M., Aspin, C., \& McLean, I. S. 1990, ApJ, 356, 544 